\section{Extensive catastrophic thromboses from elevation of factor VIII}

\author{
Jacqueline Kropf, ${ }^{1}$ Sarah Cheyney, \\ Josselin Vachon, ${ }^{1}$ Philip Flaherty, ${ }^{2}$ \\ Mai Vo, ${ }^{2}$ Steve J. Carlan ${ }^{3}$ \\ ${ }^{1}$ Department of Internal Medicine; \\ ${ }^{2}$ Division of Critical Care Medicine; \\ ${ }^{3}$ Division of Academic Affairs and \\ Research, Orlando Regional Healthcare, \\ Orlando, FL, USA
}

\begin{abstract}
Catastrophic thrombotic syndrome, otherwise known as thrombotic storm (TS) is an extreme prothrombotic clinical syndrome that presents as rapid onset of multiple thromboembolic events affecting a large variety of vasculature. In recent studies, there has been a correlation of high plasma levels of factor VIII with thrombotic events. We present the case of a young man who exhibited multi-organ failure due to thrombotic storm. A 38-year-old male presented to the emergency department for progressive dyspnea and was diagnosed to have pulmonary embolism. The patient developed respiratory distress requiring intubation and was diagnosed with both an ST-elevation myocardial infarction and right cerebral infarction during the hospital course. The patient expired and autopsy revealed the cause of death to be myocardial, cerebral and renal infarction from widespread vascular thrombosis. Autopsy revealed cause of death to be elevated factor VIII associated thrombotic coagulopathy. Factor VIII level upon autopsy was 375\% (55$200 \%$ ). Although TS is rare, it can be lifethreatening if not recognized early. Survival depends on the prompt initiation and duration of anticoagulation.
\end{abstract}

\section{Introduction}

Catastrophic thrombotic syndrome, otherwise known as thrombotic storm (TS) is an extreme prothrombotic phenotype that presents as rapid onset of multiple thromboembolic events. ${ }^{1,2}$ Both arterial and venous vasculature can be involved and can include unusual sites, such as cerebral venous sinus and intra-abdominal vessels. ${ }^{2}$ The thrombotic storm phenotype involves five key components: underlying hypercoagulable state, a provoking factor, rapid onset, necessitating early antithrombotic therapy, and a good long term prognosis if the cycle of thromboembolism could be interrupted early. ${ }^{1}$

The coagulation cascade is a tightly regulated system affected by both pro- and anticoagulant factors leading to hemostasis. ${ }^{3}$ Factor VIII is a procoagulant factor that plays an important role in the coagulation cascade by acting as a cofactor for activated factor IX. ${ }^{3}$ In more recent studies, there has been a correlation of high plasma levels of factor VIII with thrombotic events. ${ }^{3}$ Here we present the case of a 38-year-old-male who exhibited multi-organ failure as a result of thrombotic storm in the setting of isolated factor VIII elevation diagnosed upon autopsy.

\section{Case Report}

A 38-year-old male with no significant past medical history presented to the emergency department for progressive dyspnea that had been worsening over the past 6 months. He reported the breathing difficulty initially started during exercise at the gym, but had progressed to dyspnea at rest. He denied tobacco use, anabolic steroid use, recent long-distance travel or personal history of blood clots. His family history was positive for pulmonary embolism in his uncle, at an unspecified age. His review of systems was positive for two episodes of recent vomiting as well as a severe headache.

Upon arrival, he was tachycardic with a rate of 115 , normotensive $(133 / 85 \mathrm{mmHg})$, normal respiratory rate, with an oxygen saturation of $95 \%$ on room air. Laboratory results revealed a normal complete blood count (CBC) and normal basic metabolic profile (BMP) with the exception of mild creatinine elevation to $1.34 \mathrm{mg} / \mathrm{dL}(0.70-1.30$ $\mathrm{mg} / \mathrm{dL}$ ). N-terminal pro-B-type natriuretic peptide (BNP) was elevated to $294 \mathrm{pg} / \mathrm{mL}$ (0-100 pg/mL) and troponin I was 0.34 $\mathrm{ng} / \mathrm{ml}$. Electrocardiogram (EKG) revealed $\mathrm{T}$ wave inversions suggestive of anterior and inferior wall ischemia. Computed tomography angiography (CTPA) of thorax was performed and revealed bilateral pulmonary embolism with thrombus in the distal right and left main pulmonary arteries extending into the upper and lower multiple segmental pulmonary arteries. Right heart strain was also indicated by a right ventricular to left ventricular (RV/LV) ratio of 2.0. The patient was started on intravenous (IV) heparin drip with institutional anti-Xa monitoring parameters and admitted to critical care medicine for further work up.

While in the cardiac Intensive Care Unit (ICU), the patient underwent echocardiogram $(\mathrm{EKG})$ which revealed ejection frac-
Correspondence: Steve J. Carlan, Division of Academic Affairs - NOT Critical Care, Orlando Regional Healthcare, 1401 Lucerne Terrace, 2nd floor, Orlando, 32806 FL, USA. Tel.: +1.407.841.5297 - Fax: +1.321.481.0182.

E-mail: stevecarlan@gmail.com

Key words: Catastrophic thrombosis; factor VIII; arterial thrombosis; venous thrombosis; pulmonary embolus.

Contributions: JK, study design, data collection, manuscript preparation, and literature search; SC, data interpretation and manuscript preparation; JV, MV, manuscript preparation and literature search; PF, data collection and literature search; SJC, data collection, manuscript preparation, and literature search.

Conflict of interests: the authors declare no conflict of interests.

Availability of data and materials: Patient data is available subject to privacy policies and laws.

Ethics approval and consent for publication: Case report are not considered 'research' and are therefore exempt from usual IRB application and review. The patient expired and no consent could be obtained.

Received for publication: 12 May 2020.

Revision received: 14 August 2020.

Accepted for publication: 25 August 2020.

This work is licensed under a Creative Commons Attribution NonCommercial 4.0 License (CC BY-NC 4.0).

(C) Copyright: the Author(s), 2020

Licensee PAGEPress, Italy

Clinics and Practice 2020; 10:1265

doi:10.4081/cp.2020.1265

tion (EF) of 55-59\% with severe tricuspid valve regurgitation, elevated right ventricular systolic pressure is $76 \mathrm{mmHg}$ and severe pulmonary hypertension. Later that evening, the patient became acutely hypoxic and was intubated for acute hypoxic respiratory failure. EKG revealed ST segment elevations in leads II, III and AVF indicating an inferior wall myocardial infarction and was taken for emergent cardiac catheterization. He was found to have an occlusion of the right coronary artery (RCA), which was removed by mechanical thrombectomy system (Penumbra). Further work up included bilateral venous duplex of the lower extremities, which revealed a deep vein thrombosis of the right popliteal vein.

Later that evening, the patient had a decline in neurological status. His pupils became fixed and non-reactive, measuring $5 \mathrm{~mm}$ on the right and $4 \mathrm{~mm}$ on the left. A computed tomography (CT) head revealed a large right-sided evolving middle cerebral artery (MCA) territory infarct, large new left pos- 
terior cerebral artery (PCA) territory infarction, extensive edema, midline shift to the right approximately $11.3 \mathrm{~mm}$ with subfalcine herniation. Subsequent CTA of the head and neck revealed there were large areas of infarct in right MCA territory and left PCA territory and extensive occlusion of left common carotid artery at the aortic arch origin extending throughout the common carotid artery and into the external carotid artery. An arterial duplex of all four extremities showed occlusion of the left anterior tibial artery and left-sided proximal and distal brachial and radial arterial thrombus. The partial thromboplastin time (PTT) was normal at 28 seconds (26.2-37.2 s) and prothrombin time was slightly prolonged at $16.1 \mathrm{~s}$ (9.5-13.3 s). His fibrinogen was slightly elevated at $484 \mathrm{mg} / \mathrm{dL}(197-448$ $\mathrm{mg} / \mathrm{dL}$ ), inconsistent with disseminated intravascular coagulation (DIC). Heparin was therapeutic based on anti-Xa monitoring, with a range of $0.32-0.78 \mathrm{IU} / \mathrm{mL}(0.30$ $0.70 \mathrm{IU} / \mathrm{mL}$ ). There was concern for catastrophic antiphospholipid antibody syndrome, and the patient was started on stress dose steroids.

Stroke neurology was consulted and recommended magnetic resonance imaging (MRI) brain. MRI of the brain without contrast revealed a large right MCA and left PCA infarcts with additional smaller infarcts bilaterally and above the tentorium consistent with embolic disease. (Figure 1) The patient was started on hypertonic saline, placed on a continuous electronecephalogram (cEEG) and given seizure prophylaxis with levetiracetam $1000 \mathrm{mg}$ twice a day. Neurosurgery and vascular surgery were

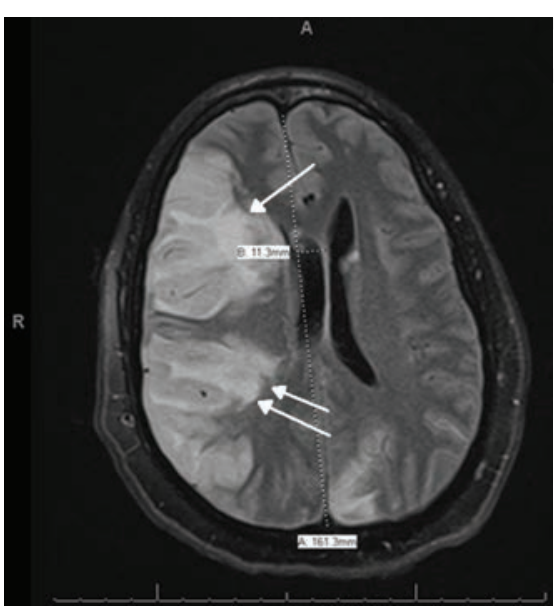

Figure 1. Magnetic resonance imaging of the brain shows large showing right middle cerebral artery (single arrow) and left posterior cerebral artery (double arrow) infarcts with additional smaller infarcts bilaterally and above the tentorium consistent with embolic disease. both consulted for possible surgical intervention. Due to the extent of thrombotic burden and severity of symptoms, no intervention was recommended. The decision was made between neurology, neurosurgery, and vascular surgery to discontinue the heparin drip due to high risk of hemorrhagic conversion.

The workup for antiphospholipid syndrome, including beta 2 glycoprotein, cardiolipin, lupus anticoagulant and PS/PT IgG was negative. Further extensive hematologic workup was also negative, including rheumatoid factor, ANA, homocysteine, factor $\mathrm{V}$ mutation, factor II mutation, lipoprotein A, and antithrombin III (ATIII). Protein $\mathrm{C}$ was low at $50 \%(70-150 \%)$, and protein S was normal, 138\% (55-165\%). Protein factor VIII assay was elevated to $375 \%(55-200 \%)$.

On the fifth day of admission the patient expired. Autopsy was performed. The cause of death was myocardial, cerebral and renal infarction from widespread vascular thrombosis secondary to elevated factor VIII

Figure 1. Magnetic resonance imaging of the brain shows large showing right middle cerebral artery (single arrow) and left posterior cerebral artery (double arrow) infarcts with additional smaller infarcts bilaterally and above the tentorium consistent with embolic disease.

associated thrombotic coagulopathy.

\section{Discussion}

The diagnosis of thrombotic storm is made based on clinical presentation. ${ }^{4}$ Because there is no specific test, the diagnosis can be difficult and therefore delayed. Clinical diagnostic criteria for TS include: acuity, location, and age of the patient. ${ }^{5}$ Location refers to thrombotic events in unusual locations, including hepatic, cerebral, portal system, and skin. ${ }^{5}$ The arterial circulation is typically involved and patients can present with stroke and myocardial infarction. ${ }^{1}$ Criteria may also include an abnormal response to therapy or the presence of an initiating event. ${ }^{5}$ While some studies cite inflammation, cocaine use, infections, trauma, and surgery as possible triggers for a thrombotic storm, a clear link has not been established. ${ }^{5}$

The patient described in this case exhibited clear evidence for TS, which resulted in myocardial, cerebral and renal infarction. Thorough investigation into potential underlying hypercoagulable was performed. Autopsy did not reveal any signs of malignancy to suggest cancer-associated thrombosis. Considerably elevated factor VIII of $375 \%(55-200 \%)$ was the only abnormality found during blood work and upon autopsy.

Factor VIII (FVIII) is a protein that acts as a cofactor for activated factor IX in the coagulation cascade. FVIII is produced by the liver, spleen, and lymph nodes. Factor VIII is a procoagulant responsible for activating factor $\mathrm{X}$ which generates thrombin. Thrombin then forms stable fibrin resulting in a blood clot. ${ }^{4}$ While studies show a correlation of elevated factor VIII with thrombotic events, our case depicts a severe care of TS due to elevated factor VIII associated thrombotic coagulopathy. ${ }^{6}$

Numerous studies have described not only a dose-dependent relationship between factor VIII and venous thromboembolism (VTE) but also an increased risk of recurrent VTE in patients with elevated factor VIII. ${ }^{3}$ A FVIII level of $>150 \mathrm{IU} / \mathrm{dL}$ is associated with a 6-fold increased risk for VTE compared to a FVIII level less that $100 \mathrm{IU} / \mathrm{dL} .^{6}$ With every $10 \mathrm{uL} / \mathrm{dL}$ increase of factor VIII, the risk of a first thrombotic event increases by $10 \% .^{7}$ Our patient had a $375 \%(0-200 \%)$ increase in factor VIII, which significantly increased the risk of thrombosis. The mechanism of FVIII elevation leading to VTE is not well understood. It has been suggested that the elevated factor can alter the delicate balance of coagulation and initiate the formation of thrombin or alter the rate of thrombin formation. ${ }^{6,7}$

While elevated levels of factor VIII are known to be a risk factor for VTE, reports also show an association with increased risk of arterial thromboembolism. ${ }^{4,7}$ The association between factor VIII and coronary artery disease has been depicted in several case-control studies. ${ }^{7}$ Previous studies have also established that patients with elevated factor VIII are more predisposed to acute ischemic strokes. Also, the severity of acute ischemic strokes based on the NIH Stroke Scale/Score has also been determined to be directly proportional to elevation in factor VIII. ${ }^{8}$ Therefore, elevated factor VIII is a considerable risk factor for both arterial and venous thrombosis but with a greater influence on venous as opposed to arterial. ${ }^{9}$

As of yet, no genetic variations have been implicated as a possible cause for elevated factor VIII levels. ${ }^{9}$ Though acute stress and chronic inflammation has been show to increase factor VIII levels, studies have shown that risk of venous thromboembolism due to elevated levels of factor VIII is independent of those caused by acute phase reactions. ${ }^{5,9}$ Treatment options for TS are aimed at treating the underlying cause, including cessation of inciting medication or initiation of steroids if CAPS is the cause. ${ }^{1}$ Plasma exchange may be indicated in CAPS or in atypical TTP. ${ }^{1}$ Regardless of 
the underlying pathology, initiating prompt anticoagulation is key to survival. ${ }^{1}$ Though the patient illustrated here was placed on immediate IV anticoagulation and steroids, extensive and catastrophic thrombotic events continued.

\section{Conclusions}

Although thrombotic storm is rare, its lifethreatening risk warrants the need for early diagnosis as well as differentiation from other catastrophic thrombotic syndromes. While recent studies have shown a correlation of high plasma levels of factor VIII with thrombotic events, our case depicts catastrophic vascular thrombosis due to elevated factor VIII associated thrombotic coagulopathy. Treatments can vary significantly between different causes of TS, and misdiagnosis can result in treatment delay and increase the risk of adverse outcomes. ${ }^{5}$ Treatment typically involves immediate, continuous, and therapeutic anticoagulation. Interrupted anticoagulation exacer- bates thrombosis cascade due to new clots containing and secreting thrombin. ${ }^{5}$ The survival of a patient with TS is dependent on duration of anticoagulation therapy. ${ }^{5}$

\section{References}

1. Lasek-Bal A, Puz P, Kazibutowska Z. Elevated factor VIII level and stroke in patients without traditional risk factors associated with cardiovascular diseases. Neuropsychiatr Dis Treat 2013;9:84752.

2. Rietveld IM, Schreuder M, Reitsma PH, Bos MH. Elevated coagulation factor levels affect the tissue factor-threshold in thrombin generation. Thromb Res 2018; 172:104-9.

3. Ortel TL, Kitchens CS, Erkan D, et al. Clinical causes and treatment of the thrombotic storm. Expert Rev Hematol 2012;5:653-9.

4. Rana MA, Mady AF, Lashari AA, et al. Lethal end of spectrum of clots-thrombotic storm. Case Rep Crit Care
2018;2018:1-6.

5. Kamphuisen PW, Eikenboom JCJ, Bertina RM. Elevated Factor VIII levels and the risk of thrombosis. Arterioscler Thromb Vasc Biol 2001;21:731-8.

6. Tóth NK, Székely EG, Czuriga-Kovács $\mathrm{KR}$, et al. Elevated factor VIII and von Willebrand factor levels predict unfavorable outcome in stroke patients treated with intravenous thrombolysis. Front Neurol 2018;8:1-10.

7. Jenkins PV, Rawley O, Smith OP, Odonnell JS. Elevated factor VIII levels and risk of venous thrombosis. $\mathrm{Br} \mathrm{J}$ Haematol 2012;157:653-63.

8. Ortel TL, Erkan D, Kitchens CS. How I treat catastrophic thrombotic syndromes. Blood 2015;126:1285-93.

9. Chandler W, Rodgers GM, Sprouse JT, Thompson AR. Elevated hemostatic factor levels as potential risk factors for thrombosis. Arch Pathol Lab Med 2002;126:1405-14. 